Questions vives

\section{Questions Vives}

Recherches en éducation

$\mathbf{N}^{\circ} 32 \mid 2019$

L'accueil et la formation linguistique des migrants. Quelles pratiques aujourd'hui?

\title{
Ingénierie didactique coopérative : quelle pratique de collaboration entre professeurs et chercheurs?
}

Conditions pour la constitution d'une expérience collective

Cooperative didactic engineering and its collaborative practices between

teachers and researchers. The conditions of a collective experience.

\section{Mireille Morellato}

\section{(2) OpenEdition \\ 12 Journals}

\section{Édition électronique}

URL : https://journals.openedition.org/questionsvives/4438

DOI : $10.4000 /$ questionsvives. 4438

ISSN : $1775-433 \mathrm{X}$

Éditeur

Université Aix-Marseille (AMU)

\section{Édition imprimée}

Date de publication : 30 décembre 2019

ISBN : 978-2-912643-56-8

ISSN : $1635-4079$

\section{Référence électronique}

Mireille Morellato, «Ingénierie didactique coopérative : quelle pratique de collaboration entre professeurs et chercheurs ? ", Questions Vives [En ligne], № 32 | 2019, mis en ligne le 30 octobre 2020, consulté le 21 septembre 2021. URL : http://journals.openedition.org/questionsvives/4438; DOI : https://doi.org/10.4000/questionsvives.4438

Ce document a été généré automatiquement le 21 septembre 2021.

\section{cc) $($ ) $\ominus$}

Questions Vives est mis à disposition selon les termes de la licence Creative Commons Attribution -

Pas d'Utilisation Commerciale - Pas de Modification 4.0 International. 


\section{Ingénierie didactique coopérative : quelle pratique de collaboration entre professeurs et chercheurs?}

Conditions pour la constitution d'une expérience collective

Cooperative didactic engineering and its collaborative practices between teachers and researchers. The conditions of a collective experience.

Mireille Morellato

\section{Contexte : l'ingénierie didactique coopérative « Ace- Arithmécole »}

1 Nous cherchons à décrire la coopération entre des professeurs des écoles et des chercheurs dans le cadre d'une ingénierie didactique coopérative (Sensevy, Forest, Quilio, \& Morales, 2013) nommée «Ace-Arithmécole». Cette ingénierie porte sur la construction des nombres pour des élèves de cycle 2 (élèves de 6 à 8 ans) et couvre tout le programme scolaire français en numération, calcul et résolution de problèmes. Elle s'appuie sur des résultats de la recherche en didactique des mathématiques et en sciences cognitives ${ }^{1}$. Après avoir brièvement rappelé ce que nous entendons par « ingénierie didactique », nous préciserons en quoi cette ingénierie didactique est dite coopérative.

2 Une ingénierie didactique est un dispositif conçu par et pour la recherche (Artigue, 1990). Il permet l'étude empirique de phénomènes d'enseignement-apprentissage, artificiellement produits et observés, afin de les modéliser. Les mises en œuvre de situations didactiques en constituent le dispositif expérimental. Guy Brousseau a mis en place un tel dispositif au COREM ${ }^{2}$ entre 1972 et 1997. Denise Greslard et Marie-Hélène Salin ont participé en tant qu'enseignante pour la première et chercheure pour la seconde aux ingénieries didactiques développées au COREM. Elles témoignent d'une collaboration essentielle pour la mise à l'épreuve d'une ingénierie didactique (Greslard 
\& Salin, 2011). Mais le travail de conception de la séance y était de la responsabilité du chercheur qui devait ensuite l'expliciter, le clarifier pour que l'enseignant puisse mettre en œuvre la séance.

3 Puisque la didactique est la science d'une pratique d'enseignement (Sensevy \& Mercier, 2007), elle est nécessairement confrontée aux conceptualisations et aux actions des professeurs. Il faut alors, selon ces auteurs, l'examiner au sein de l'action effective, en relation avec le savoir, du professeur et de ses élèves dans leur classe. Il ne s'agit pas pour autant de réduire cette science à l'explicitation des pratiques des enseignants. Une orientation de travail est ainsi donnée :

Pour mettre en travail cette différence - pour une part irréductible - entre sémantique familière de l'action et langage des modèles, il existe donc tout un système pratique à élaborer et promouvoir, celui de recherches coopératives, dans lesquelles la production d'ingénierie didactique d'un nouveau genre se fasse sous la responsabilité conjointe des professeurs et des chercheurs, construisant ensemble des fins communes à un processus pensé comme collectif, au sein de dispositifs qui restent pour la plus grande part à inventer (ibid., p. 209).

Dans l'ingénierie de la recherche Ace-Arithmécole, les séquences d'enseignement sont conçues, à la fois par des professeurs et des chercheurs. Leur mise en œuvre est analysée collectivement et les modifications apportées sous la responsabilité de tous. Le dispositif a été implémenté dans une soixantaine de classes ${ }^{3}$, dites expérimentales, en 2012-13. Depuis, chaque année, d'autres classes rejoignent le dispositif. Cette ingénierie poursuit une double direction :

i. mener un travail théorique sur les situations didactiques et le savoir en jeu (le collectif professeurs/chercheurs se questionne sur un contenu de savoir et sa mise en œuvre afin qu'ils soient efficaces);

ii. s'adapter aux contraintes de l'enseignement ordinaire (les situations didactiques doivent être utilisables pour, à terme, pouvoir être diffusées dans des classes ordinaires, hors dispositif de recherche).

5 Nous étudions dans ce qui suit comment des professeurs et des chercheurs coopèrent dans une telle organisation collective pour constituer cette ressource d'enseignement. De quoi est constitué leur travail commun? Que dit-il de cette pratique sociale de collaboration?

\section{Le dialogue d'ingénierie comme institution pour la constitution d'une expérience collective}

Pour que les professeurs et les chercheurs puissent concevoir ensemble une ressource, un espace dialogique est créé, sous la forme de réunions régulièrement tenues. Nous pensons que cet espace dialogique est une institution de nature coopérative, pivot de l'articulation didactique entre l'individuel et le collectif. En effet, chaque membre du groupe de recherche va vivre une expérience commune, celle de la mise en œuvre de l'ingénierie. Ni les uns, ni les autres ne savent a priori comment elle se déroulera.

7 À partir d'un document décrivant les situations didactiques ${ }^{4}$, les professeurs des classes expérimentales procèdent à une mise en œuvre dans leur classe. Ce sont ces mises en œuvre qui sont rapportées et discutées lors de réunions, avec l'appui de comptesrendus de séance et d'une liste de diffusion. Au cours de ce procès de la mise en œuvre, le travail du collectif contribue à objectiver les connaissances produites. 
8 Ainsi les réflexions peuvent devenir des expériences vécues partagées, ou faits d'expérience (Fleck, 2008). Ces faits concourent à la constitution de "voir comme" communs, c'est-à-dire des concepts et des pratiques partagés au sein d'une communauté. Autrement dit d'un même style de pensée (ibid.) nécessaire à la production d'échanges scientifiques pour une action commune.

Nous étudions dans cet article certaines des conditions sous lesquelles une construction commune d'un tel état d'expérience peut se mettre en place.

\section{Cadre théorique}

Nous nous appuierons sur la théorie de l'action conjointe en didactique (Sensevy, 2011; Sensevy \& Mercier, 2007) car cette théorie permet de décrire le dialogue d'ingénierie comme un lieu de transactions. En effet, chacun a la possibilité de prendre en compte l'action d'autrui en coordonnant ou ajustant son action en fonction de celle d'autrui. Il ne s'agit pas d'œuvrer ensemble mais d'œuvrer conjointement à la diffusion de savoirs. L'action de l'un dépend de, se réfère à celle de l'autre par rapport à un objet de savoir. Les transactions sont alors vues comme une enquête (Dewey, 1993) des professeurs et chercheurs sur un savoir ingénierique (que nous préciserons par la suite) dans un environnement sur lequel ils agissent.

11 Cet environnement est caractérisé par un contrat et un milieu. Le contrat est constitué d'un système de connaissances disponibles, individuelles et collectives, antérieures à la mise en œuvre de l'ingénierie ou issues du début de cette mise en œuvre. Ce système est actualisé par des problèmes soulevés par la mise en œuvre. Ces problèmes se constituent alors en faits à questionner selon des nécessaires et des possibles pour l'action coopérative. Le milieu fait problème pour tous les professeurs, mais aussi pour les chercheurs, puisqu'il est constitué par la mise en place d'une séquence d'enseignement-apprentissage, en cours d'élaboration. Cette séquence véhicule, de plus, des présupposés et des hypothèses d'ingénieries spécifiques. Ainsi tous sont amenés à élaborer le milieu de l'enquête.

\section{Un exemple emblématique de dialogue d'ingénierie entre professeurs et chercheur}

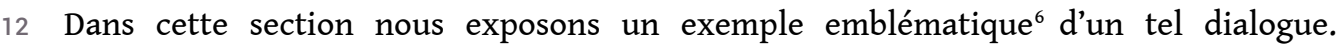
L'exemple ici n'est pas une illustration du propos mais une manière de rendre compte concrètement du travail réalisé. Il est emblématique pour deux raisons principales : (i) il exemplifie, nous verrons ci-dessous comment, certaines des conditions essentielles de la constitution de l'expérience collective; (ii) il montre une pratique d'analyse et du positionnement épistémologique de notre travail dans sa force à expliciter, enrichir, questionner, "dire". Nous le mettons au travail afin que soit décrite la coopération professeurs / chercheurs, ses finalités et ses effets dans la pratique. 


\section{Ce qu'il faut savoir de la situation didactique proposée aux élèves pour comprendre l'arrière-plan des transactions}

\section{Présentation de la situation didactique proposée aux élèves}

La coopération entre professeurs et chercheurs est fondée sur le développement pour l'enseignement d'une situation didactique de base, nommée "Jeu des annonces" ». Nous allons la décrire précisément dans ce qui suit. Cette situation est proposée aux élèves pour qu'ils y découvrent et construisent un savoir. Le savoir visé, à terme, est la compréhension de la numération décimale positionnelle. Ce qui se joue, dans les premiers temps, avec du matériel (des doigts et des dés) sur de " petits » nombres de 0 à 12 , se travaillera par la suite et à l'écrit avec des nombres plus grands.

Le jeu des annonces est une situation de désignation et de comparaison de mesures. Les élèves expriment la mesure d'une grandeur, ici la taille d'une collection de points ou de doigts, puis comparent les deux mesures (nombres de points et de doigts). L'un de ces nombres est obtenu par le lancer d'un dé. L'autre choisi par l'élève avec les doigts de ses deux mains qu'il montre, comme l'illustre la figure 1.

Figure $1:$ Une partie de jeu des annonces

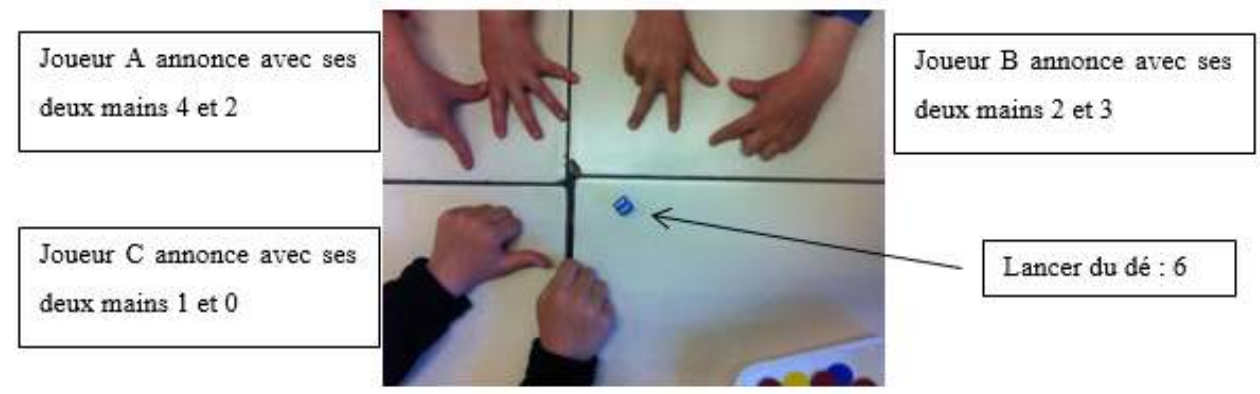

Les doigts immobilisés sont montrés avant le lancer du dé, à la manière d'un pari sur ce qui va sortir. «L'annonce » des doigts devra être comparée avec « le lancer ». Le jeu est gagné si l'annonce est égale au lancer. Le joueur A pourra dire: «j'ai gagné, mon annonce 4 et 2 est équivalente au lancer 6 ». Le joueur B pourra dire : «j'ai perdu, 3 et 2 ne sont pas comme $6 »$. Plus tard, ils exprimeront par écrit le gain ou la perte au jeu de comparaison: $« 4+2=6 » ; 《 3+2 \neq 6 »$ ou $« 3+2<6 »$.

Cette situation didactique fonde ainsi le travail des élèves sur des comparaisons d'écritures additives. La référence aux annonces se construira peu à peu en paradigme pour les élèves. En effet, le jeu des annonces va se répéter tout en évoluant sur un grand nombre de séances pour former un ensemble de situations. Le nombre et la valeur des termes augmenteront. Ainsi, de nouvelles questions se poseront; questions relatives à la comparaison d'écritures additives et à son expression écrite mais aussi à la désignation écrite des nombres.

\section{Sujet de la discussion entre professeurs et chercheur, relatif à une phase de ce jeu}

Les protagonistes des échanges que nous allons analyser sont des professeurs des écoles de cours préparatoire (CP) et un chercheur d'un groupe local ${ }^{7}$. Ces échanges ont lieu lors de la première année d'implémentation du dispositif dans des classes 
expérimentales, en 2012-13. Le sujet que ce collectif va aborder porte sur une nouvelle étape de la progression. La comparaison entre deux écritures additives amène à construire la notion de différence. La différence est ici considérée comme l'écart entre deux mesures. Un des principes mathématiques sur lesquels repose la démarche d'enseignement-apprentissage des nombres dans Ace-Arithmécole est le développement d'un rapport pré-algébrique aux mathématiques (Artigue, 2011; Carraher \& Schliemann, 2014). Le travail sur le principe inverse (Verschaffel, Bryant, \& Torbeyns, 2012) y tient une place centrale. Les élèves apprennent à associer écritures additive et soustractive (par exemple, $5=2+3$ donc $5-3=2$ ). Habituellement, dans l'épistémologie pratique des professeurs (Sensevy, 2007), la différence est considérée comme ce qu'on enlève, ôte, retire dans une opération concrète sur des objets. Comment cette notion va-t-elle être abordée puis discutée au sein de ce collectif ? Que nous disent ces échanges de la nature de la coopération entre chercheurs et professeurs?

\section{Eléments de méthodologie}

Pour mettre en évidence la dimension dialogique des échanges, nous nous sommes appuyée sur le logiciel Transana (Fassnacht \& Woods, 2001), logiciel d'aide à la transcription, à la gestion des données et à l'analyse des données filmées ${ }^{8}$. Nous avons procédé d'abord à un filmage des réunions qui constituent nos données principales. Puis nous avons transcris l'intégralité des échanges. Nous avons découpé ce corpus en épisodes qui mettent en évidence des unités de sens dans le flot de la discussion. Nous avons ensuite opéré des sélections d'épisodes en établissant des requêtes dans la base de données du logiciel. Ces requêtes permettent d'obtenir des collections d'épisodes, grâce au moteur de recherche du logiciel, à partir de mots-clés attribués à chacun des épisodes. Nous avons ensuite procédé à une réduction des épisodes d'une collection en une vue synoptique (Wittgenstein, 2004) pour constituer chacune des séries épisodiques.

Une vue synoptique est une forme de compréhension des phénomènes qui met au jour des éléments existants en les disposant de telle manière que se dévoilent des relations susceptibles de produire des connaissances (Sensevy, 2011). Le travail de réduction des données recueillies transforme ce qui est observable et représentable pour produire « un arrangement possible [des objets de l'empirie] en vue de les penser» (Glock, 2003, p. 585).

Une série épisodique est donc constituée d'épisodes signifiants, relatifs à une pratique de coopération concernant l'étude de l'objet didactique à mettre en œuvre dans la séquence d'enseignement. Nous allons nous appuyer sur une telle série pour décrire les transactions dans le cadre de l'ingénierie didactique coopérative Ace-Arithmécole, en nous centrant sur les conditions de cette coopération. Nous associerons ensuite la série constituée avec une observation réalisée dans une classe.

\section{Une série épisodique comme exemple emblématique des conditions de la coopération}

Obtention de la série épisodique

Nous exposons ici des moments d'échanges entre des professeurs des écoles et un chercheur. Ils concernent l'introduction de la notion de différence et sa mise en place 
effective dans la classe. Ils ont lieu durant la première année d'expérimentation (2012-13), lors des septième et huitième réunions sur les dix prévues par le dispositif. Les échanges relatifs à la différence sont conséquents car ils se déroulent sur une durée de près de quatre heures sur les cinq heures trente effectives des deux réunions. La notion de différence sera à nouveau discutée dans la neuvième réunion ${ }^{9}$, puis lors des années suivantes de mises en œuvre expérimentales (2013-14 et 2014-15). La constitution d'une expérience partagée et d'un style de pensée relatifs à cette notion telle qu'elle est mise en place dans l'ingénierie a nécessité une très longue durée. Cependant, dans le cadre de cet article, nous ne traiterons pas des échanges dans leur dimension diachronique à cette échelle temporelle de plusieurs années, mais à une échelle plus réduite.

La recherche documentaire que nous avons entreprise avec Transana nous a permis de dénombrer 76 épisodes avec les mots-clés " différence » et " réunion du 6 mars 2013 » (septième réunion), « réunion du 20 mars 2013 » (huitième réunion). Un grand nombre de ces épisodes contiennent d'autres mots-clés. Ceux-ci montrent les liens sémantiques entretenus dans la discussion ${ }^{10}$.

\section{Présentation de la série épisodique}

Lors de la septième réunion du 6 mars 2013, le chercheur (désigné par « $\mathrm{CH} »)$ propose de réfléchir sur le module consacré au travail de la différence entre deux mesures. Voici comment il s'adresse aux professeurs :

L'idée c'est de réfléchir collectivement à comment ça a été réalisé, de voir éventuellement ce qui correspond plutôt à ce que vous, personnellement, vous aviez envisagé de faire, comment vous aviez compris les choses. Parce que là il y a des phénomènes qui vont apparaître, qui vont nous paraître pertinents, pas pertinents mais c'est pas, vous comprenez, c'est pas de dire : c'est pas bien, c'est pas du tout ça. Mais c'est de faire ce travail d'observation comme nous on le fait dans la recherche. On va le mener collectivement afin de voir comment ce module peut être mis en œuvre de la façon la plus productive possible. C'est pas une solution qui va apparaître mais plusieurs choix possibles avec des options. $(\mathrm{CH}$, Tour de paroles 20)

Ce nouveau module fait suite aux modules consacrés au jeu des annonces qui se sont déroulés sur un temps long de six mois. Après le jeu avec mains et dés, les comparaisons d'annonces se sont réalisées avec des nombres : deux termes comparés à deux termes, puis $\mathrm{x}$ termes à $\mathrm{y}$ termes. Pour permettre la comparaison de telles «longues» écritures additives, les élèves ont dû éprouver la nécessité de regroupements stratégiques de termes. En effet, ils ne maîtrisent pas toujours le calcul d'une somme comportant un "grand» nombre de termes. Ces regroupements sont basés sur des résultats acquis par l'expérience du jeu et des techniques de composition et décomposition.

Dans ce nouveau module de la différence, il ne s'agit plus de comparer des annonces mais de désigner une différence entre deux annonces. Désigner la différence entre deux annonces enrichit la description de leur comparaison. Ainsi l'ensemble des situations didactiques proposées se caractérise par un principe de continuité et de rupture défini comme un temps de situation (Quilio, 2008; Sensevy, 2011). L'avancée du temps didactique « repose sur la mise en perspective d'une situation initiale fondatrice par la production d'une référence évolutive" (Quilio, 2012, p. 24). Mener une progression 
selon un temps de situation est une pratique didactique peu habituelle; elle nécessite une analyse collective.

Lors de la réunion suivante, les professeurs font un inventaire des modalités choisies lors de la mise en œuvre, des ajustements réalisés, des constats sur les difficultés rencontrées afin que soient prises des décisions collectives pour modifier la proposition faite dans le document support de l'ingénierie.

Nous présentons dans la section suivante une série épisodique sur la constitution d'un fait d'expérience relatif à la mise en œuvre effective du module. Pour maintenir la lisibilité sur ce que nous pouvons dégager de l'ensemble de la série comme connaissances pour l'ingénierie, nous avons organisé la série épisodique en trois moments de deux épisodes chacun. Nous avons dû également effectuer des coupes dans les tours de paroles pour en faciliter la lecture.

\section{Description des épisodes concernant l'introduction de la notion de différence et sa mise en place effective}

Dans le premier moment de la série, lors de la septième réunion, des professeurs comparent la mise en œuvre réalisée dans leur classe à la mise en œuvre vue sur un film tourné dans une classe d'étude ${ }^{11}$. Les échanges aboutissent à un premier travail collectif sur la notion. Dans un deuxième moment, toujours lors de la septième réunion, deux professeures (Emp et Ann) proposent une mise en œuvre. Le troisième moment est extrait de la réunion suivante (huitième réunion) : les mises en œuvre évoquées ont été entre-temps travaillées dans les classes. Elles sont discutées à l'aune de cette mise en place. Nous présentons, ci-dessous, pour chacun des épisodes, les échanges puis immédiatement après (ou avant) des éléments d'aide à leur compréhension.

Premier moment de la série, septième réunion du 6 mars 2013 :

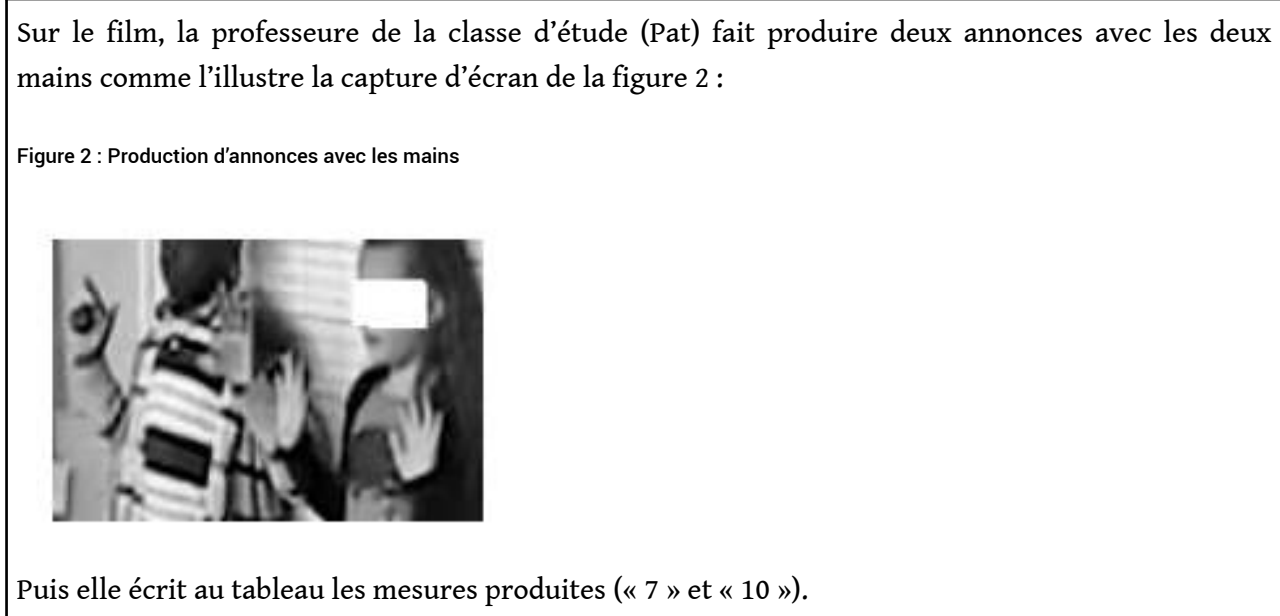


Extrait épisode $53 \min 40$ - 55 min 13 ; Tdp 67-84

Emm : elle [Pat] écrit tout de suite au tableau les nombres des annonces. Moi je n'ai rien écrit pour qu'ils comprennent bien le sens de ce que c'était une différence. Je suis pas passée par les nombres. Je suis passée par les doigts [différence entre deux annonces montrées avec les doigts] et en lien avec résolution de problèmes. Je suis passée par les immeubles, les étages, quelle est la différence [du nombre d'étages entre deux immeubles].

Kay : après avoir sorti les doigts, les enfants regardaient avec des yeux étonnés. Emm [qui est sa collègue de la classe voisine avec qui elle échange régulièrement] a essayé avec les immeubles et les trains.

Emm : ce train est plus long de combien [de wagons], quelle est la différence entre eux, combien il faudrait [de wagons] au train rouge pour atteindre le train bleu, tout ce vocabulaire là pendant la première séance.

Commentaires :

La professeur Emm fait appréhender la différence dans des situations variées (nombre de doigts levés de deux annonces, d'étages de deux immeubles, de wagons de deux trains) après avoir constaté, comme sa collègue (Kay) le précise, que les élèves ne semblaient pas comprendre ce qui était attendu à partir des seules annonces montrées. Le travail de la différence dans la classe de Emm commence par une description de la situation matérielle car les wagons du train sont représentés par des cubes (« ce train est plus long de combien, combien il faudrait au train rouge pour atteindre le train bleu »). 
Extrait épisode 56 min 26 - 1h 01 min 39 ; Tdp 119-137

$\mathrm{CH}$ : ce qui a été présenté [film classe d'étude] commence par deux annonces différentes. On convoque deux élèves et la question qui est posée c'est, en montrant avec les doigts, est-ce que a priori ces annonces sont-elles différentes ou pas?

Kay : oui elles le sont.

CH : elles le sont tel que c'est présenté. Parce que la configuration des doigts n'est pas la même. Mais pour autant les annonces sont-elles différentes au sens de ce qu'attend le prof là ? On est bien d'accord que si je fais ça (montre 5 et 2 avec ses deux mains) et si je fais ça (idem avec 4 et 3), les deux annonces sont différentes donc le problème que vont rencontrer les élèves il est de deux ordres : si je montre avec les doigts, on va montrer des configurations qui sont nécessairement différentes, pour autant comme tu le disais Flo, ça ne garantit pas qu'elles ne désignent pas le même nombre. Il va falloir savoir si je gagne parce que j'en ai plus ou pas.

Jef : la question que j'ai posée c'est qui a gagné quand ils ont fait leur annonce.

Emm : qui est le plus grand, qui a le plus de doigts

Jef : première question qui a gagné. Et après deuxième question, la différence.

Kay : de combien.

Jef : combien de plus.

$\mathrm{CH}$ : la question qu'essaie de traiter la professeure de la classe d'étude ici, c'est de construire le sens de différence. C'est comme tu le disais toi [Emm], construire le sens de différence tel que c'était proposé [dans le document] m'a amenée à utiliser autre chose : j'ai utilisé les immeubles, j'ai utilisé ...

Emm : les trains, les parcours.

CH : D'accord. Donc le problème ici, enfin la question qui est posée, pour le professeur, c'est de donner un sens à différence, à la différence MATHEMATIQUE on est d'accord ? Parce que les élèves, ils le savent bien qu'ils sont différents. Ils le voient. Mais là on a l'entrée en matière pour construire mathématiquement la notion de différence avec les annonces dans leur état initial. C'est un retour très très lointain pour les élèves, on retourne avec les doigts. D'accord tu vois bien que ça c'est 2 . Surtout ça n'utilise pas à ce stade-là des connaissances construites grâce aux doigts, c'est-à-dire les nombres. Donc pour jouer à ça, le professeur [Pat] a fait le choix de retourner à la situation initiale et de ne pas s'appuyer sur des connaissances que les élèves viennent de construire, c'est-à-dire je suis capable d'écrire une annonce sans utiliser le matériel doigts. On le voit bien ici, que le jeu qui s'installe entre le professeur et les élèves c'est un problème de relation symbolique : on a les doigts et puis on va tout de suite à un système symbolique hybride qui est la représentation sous la ligne graduée qui permet à ce moment-là pour le professeur de visualiser l'idée. On est bien d'accord, ici c'est de donner une visualisation de la notion de différence, c'est ce qu'elle [Pat] fait là. 
Commentaires :

Le chercheur attire l'attention sur la sémantique du terme « différence ». Quand on montre avec les mains les annonces « 5 et 2 » et « 4 et 3 ", ce n'est pas leur configuration "différente » qui est pertinente dans la situation, mais leur mesure (entre $5+2$ et $4+3$ la différence est de 0 ). La question de la différence est donc ici une question à traiter mathématiquement, à l'écrit ( « on a l'entrée en matière pour construire mathématiquement la notion de différence avec les annonces »). Le " retour très lointain aux doigts » évoqué pourrait laisser entendre que, pour les élèves, le travail s'effectuerait toujours sur la comparaison d'annonces (les annonces ci-dessus seraient donc équivalentes).

La notion est visualisée par la professeure Pat dans un « système symbolique hybride », entre deux lignes comme le montre la figure ci-dessous, avec la première qui représente l'annonce 9 , la seconde l'annonce 4 ; la différence 5 étant figurée par le nombre d'intervalles en plus que l'on peut compter un à un :

Figure 3 : Visualisation de la différence sur les lignes

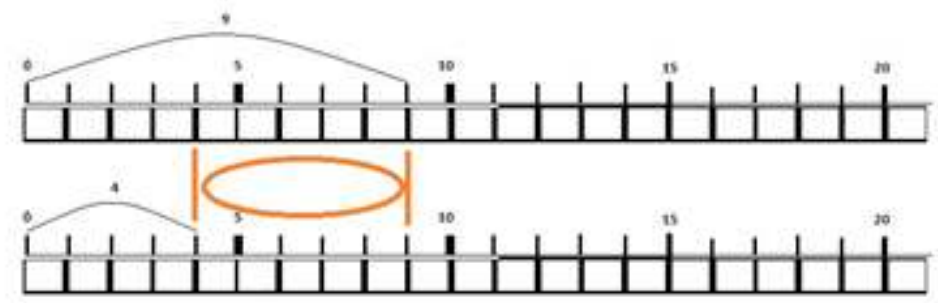

31 Ce moment montre une première analyse collective à partir d'un problème soulevé : certains élèves ne semblent pas comprendre ce qui est attendu dans la nouvelle situation. Les professeurs introduisent la différence en appui sur des situations matérialisées. Les situations proposées par les professeurs Emm et Kay sont plus variées. Les professeures utilisent le langage de la description ou en visualisent la situation sur une représentation. Nous relevons deux prises de position, qui ne sont pas forcément antagonistes: (i) introduire la notion en visualisant l'écart dans des situations réelles ou représentées (ce qui constitue une habitude partagée, un déjà-là, un contrat au sein du collectif), (ii) travailler la notion en utilisant les connaissances construites précédemment dans le jeu des annonces, sans pour autant que celles-ci soient explicitées.

Deuxième moment de la série, septième réunion du 6 mars 2013 : 
La professeur Emp rapporte une action réalisée lors du module précédent où sont comparées des annonces à x termes (ici, $8+8+2+1+10$ et $1+6+3+9+6)$.

Voici une production d'élève montrant le travail de compositions et décompositions de termes pour obtenir une comparaison entre $20+5$ et $20+9$ :

Figure 4 : Début de comparaison de longues annonces

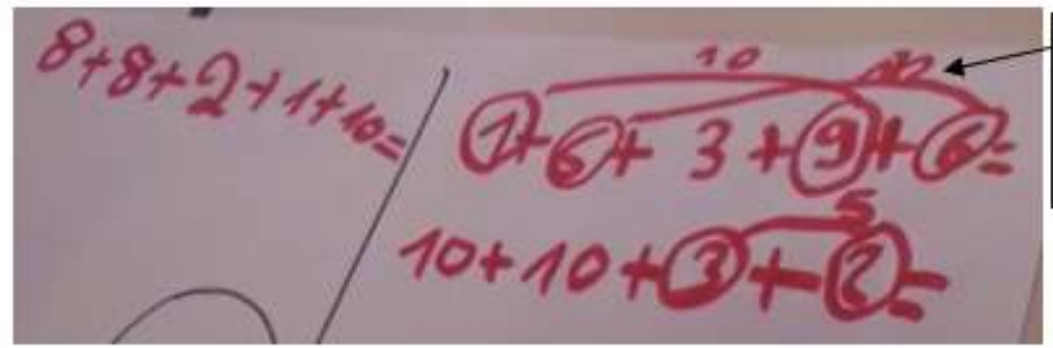

12 décomposé à la ligne en dessous en $10+2$

L'élève termine ainsi (non filmé) :

$8+10+1+1010+10+5$

$20+9>20+5$

Extrait épisode 1h 02 min 59 - 1h 04 min 42 ; Tdp 151-157

Emp: dans le module 8 [module précédent] lorsqu'on compare deux longues additions ça amène finalement à la notion de différence. On leur a posé la question : de combien la classe a gagné. Je trouvais ça beaucoup plus logique parce qu'ils comparaient des structures additives : quand on avait $20+9$ et de l'autre côté on avait $20+5$, il y a un élève qui a dit : « ben 9 là c'est $5+4$ et de l'autre côté j'ai que le 5 ». Je trouvais que ça avait beaucoup plus de sens à ce moment-là de la progression que de revenir sur les doigts.

$\mathrm{CH}$ : allons plus loin dans ta remarque : on voit bien qu'il y a matière à s'appuyer sur des connaissances qui ont été construites par les élèves, pour les solliciter, pour répondre à la nouvelle question qui est posée.

Commentaires :

En s'appuyant sur une pratique réellement mise en œuvre, Emp propose une piste mathématique pour donner sens à la différence et commencer à répondre à la nouvelle question posée (désigner la différence). Il s'agit ici de comparer in fine $5+4$ et 5 par la comparaison des termes rendus identiques (nous reviendrons sur cette expression). Il y a 4 de plus dans l'annonce $20+5+4$ que dans celle de $20+5$ ainsi décomposées. Le chercheur consonne avec cette proposition.

Extrait épisode $1 \mathrm{~h} 07$ min 53 - 1h 09 min 50 ; Tdp 180-186

Ann : hier on a comparé 8 et 9 et on a pris le terme de différence au sens premier du terme. C'est-à-dire qu'on avait 4 et 4 et on avait 5 et 4 donc on a vu qu'il y avait 4 effectivement de chaque côté et on est allé chercher le 4 dans le 5. A la fin, on avait écrit d'un côté $4+4$ et de l'autre côté $4+4+1$ : ce que tu disais un petit peu. Là, la différence, elle apparaissait comme justement ce qui n'était pas pareil. Sans parler d'enlever ou de retrancher ou de y en a en plus y en a en moins.

$\mathrm{CH}$ : parce que, et c'est là la nuance, 1 est contenu dans $4 ; 2$ est contenu dans $4 ; 3$ est contenu dans 4 . On a appris à gagner petit à petit que si on écrivait 9 , on pouvait écrire $5+4 ; 4$ est contenu dans 5 . Voilà avec toutes les formulations qu'on avait trouvées. Mais ça ça veut pas dire pour autant que la différence de 4 à 9 elle est 5 , on l'a pas encore définie.

Commentaires :

La professeure Ann affine la piste précédemment engagée par Emp en montrant comment ont été rendues identiques les termes des annonces : «on est allé chercher le 4 dans le $5 \ldots$ On a écrit $4+4$ et $4+4+1$ », sous-entendu car 8 a été décomposé en $4+4$ et 9 en $4+5$ et que l'on sait $5=4+1$. Le chercheur précise qu'il s'agit de la notion « contenu dans». 

différence. Ce point de vue a pu être concrétisé par les professeures Emp et Ann qui ont partagé leurs expériences de mises en place en classe. Celles-ci permettent de commencer à « donner chair » à la différence mathématique en la mettant en évidence par la décomposition. La décomposition est la connaissance, à la fois pratique et théorique, issue du jeu des annonces et reprise ici dans un nouvel usage. Ce deuxième moment témoigne d'une évolution de la référence aux annonces qui émerge ainsi des transactions. Une telle évolution réactualise le déjà-là partagé, celui de la différence vue comme écart dans une situation de comparaison de collections de doigts. Une telle évolution engage aussi à travailler ce déjà-là dans le milieu, en interrogeant la mise en œuvre de la notion de « contenu dans ».

\section{Troisième moment de la série, huitième réunion du 20 mars 2013 :}

Extrait épisode $35 \min 34-39 \min 50$; Tdp 113-132

Emm : mais c'est pas comme ça [le « contenu dans »] qu'on a présenté [la notion de différence dans le document de l'ingénierie]... que tout s'articule quoi !

Emp : mais avec les résolutions de problèmes un petit peu quand même.

Jef: si, si, avec les résolutions de problèmes. Avec les tours bleues, les tours rouges, c'est exactement ça. Ils égalisent en fait.

Flo : oui c'est ça, on cherche l'égalité. Combien il manque et combien il faut rajouter.

$\mathrm{CH}$ : on a un certain nombre de leviers. Effectivement là si on lit [dans le document], on n'a pas toutes les explicitations. Il va falloir qu'on fasse ce travail et c'est pour ça qu'on travaille collectivement et c'est pour ça que c'est intéressant. Mais quand même là on a les moyens d'indiquer un certain nombre de raisons pour les élèves de penser cette histoire de...

Emm : ben on n'en a jamais parlé de « contenu dans".

Plusieurs profs : oui voilà !

Emm : et c'est bien ça le souci quoi! Moi je suis persuadée que c'est ça, il faudrait qu'on s'appuie plus sur ça. Vraiment parce que ça manque. Moi mes gamins ils sont capables après de faire des soustractions mais euh si tu grattes un peu la technique et l'intuition qu'ils ont des choses, je suis persuadée que le « contenu dans » il sort pas.

$\mathrm{CH}$ : vous comprenez bien que l'écart est au cœur du problème. C'est une métaphore « est contenu dans » quand on parle de l'écart. Mais on est bien dans ce travail-là, ça avait un sens dans une certaine situation [le jeu des annonces] et puis on va donner un sens utile à la situation que l'on rencontre. Je sais pas ce que vous en pensez mais il faudrait qu'on réfléchisse à ce travail de sens. C'est un peu comme du vocabulaire: voyez un mot ou une expression peut avoir un sens, une grammaire par rapport à la situation. C'est ce travail grammatical que l'on produit. On passe de « je peux atteindre » à " est contenu dans » avec la même forme. On fait 12 en faisant $8+4$ et on écrit $12=8+4$. Mais maintenant on dit 4 « est contenu dans » 12 avec la même écriture. Pourquoi ? Parce que la situation nous amène à construire la grammaire de ça, l'arrière-plan qui donne sens à cette situation-là.

Commentaires :

L'emploi de « contenu dans » n'est pas explicité dans le document. Les professeurs Emp, Jef et Flo rapprochent le traitement mathématique possible de "contenu dans » avec les résolutions de problèmes (recherche du nombre de cubes à rajouter à la tour rouge pour atteindre la même hauteur que la tour bleue). Cependant si la notion de différence peut ainsi commencer à être appréhendée de manière concrète, son arrière-plan épistémologique n'est pas suffisamment éclairci. L'explicitation doit être menée collectivement afin de construire une grammaire de la situation adéquate, nécessaire pour passer de " je peux atteindre » à « est contenu dans » à partir de la même écriture additive. 
Extrait épisode $1 \mathrm{~h} 47 \mathrm{~min} 10$ - 1h 51 min 13 ; Tdp 502-543

Flo : le lendemain de la réunion on a fait résolution de problèmes avec la situation du train. J'en ai profité là, je me suis engouffrée dedans. J'ai demandé combien de wagons dans le train pour Marseille, combien de wagons dans le train pour Paris, il y en a 7 , il y en a 5 , quelle est la différence? Ils l'ont vue puisque c'est visible 2 [sur la représentation]. On l'a dit, j'ai écrit 5, j'ai écrit 7,j'ai écrit 2 et j'ai dit «maintenant comment on écrit la différence », ce qu'on avait vu sur un des films. Et à partir de là, à force de répéter, de comparer et d'avoir une différence qu'on voit, c'est rentré dans leur tête : vraiment quand on parle de différence c'est l'écart. Et ils ont vraiment la vision du train. A l'heure actuelle quand on fait des différences, je demande un écart de 5 , j'en ai qui sont capables d'écrire que c'est 87-82 parce qu'ils ont l'écart en fait.

Emm : mais est-ce qu'ils ont le... parce que moi je suis dans la même situation que toi, ils ont bien compris ce qui manquait. Ils ont l'addition à trous en fait. Parce que sur le schéma-ligne ça se voit bien visuellement.

Figure 5 : Le schéma-ligne

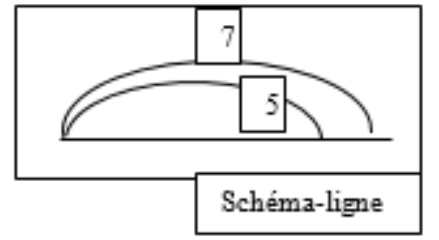

$\mathrm{CH}$ : le terme inconnu d'une somme.

Emm : mais je suis pas sûre qu'ils aient bien compris.

Flo : le contenu [le nombre contenu dans un autre]?

Emm : le contenu.

Flo : ils ont bien voilà, ils sont arrivés à comprendre l'écart...

Emm : mais pas le contenu.

Flo : j'en ai plein qui disent on part du plus petit et on cherche combien il manque pour atteindre le plus grand ; parce que je leur avais demandé comment tu fais pour écrire.

$\mathrm{CH}$ : ce que l'on peut voir dans le schéma-ligne. Toi [Flo] tu utilises le schéma-ligne et toi [Emp] tu as parlé la boîte ; ça se traduit dans le numérique par « est contenu dans ».

Flo : mais c'est ça qui pose problème.

Agn : c'est cette notion-là qui est très difficile !

Emp : et moi la boîte les recadre par rapport à ça [Emp fait désigner la différence dans la boîte, figure ci-dessous, comme une partie de la relation parties-tout, visibles dans cette représentation] :

Figure 6 : Visualisation de la différence (entourée) dans la boîte

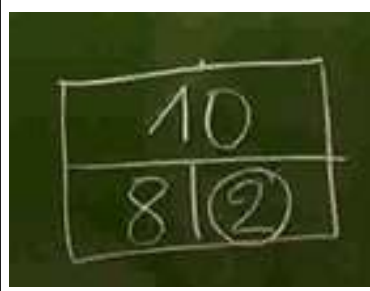

$\mathrm{CH}$ : quand tu [Flo] dis : » je suis rentrée avec le train et ça m'a aidée à faire », c'est parfait. On a une situation, on voit une propriété du nombre. Maintenant il faut que ça soit le nombre qui soit convoqué pour répondre à la situation. Donc j'ai un autre train, comment je pourrais faire? Quelle est la composition qui correspond à ça ? C'est pas le train qui va me renseigner, c'est le nombre, les propriétés que je sais sur le nombre qui vont me permettre de répondre à la solution. On sollicite ce que l'on sait sur le nombre pour répondre mais ça veut pas dire qu'on n'utilise pas la situation effective, au contraire, j'ai besoin d'une représentation, j'utilise une représentation. D'ailleurs très vite tu vas au schéma-ligne je suppose.

Flo : mais ils manipulent pas...[dans le sens de compter les intervalles sur le schéma-ligne] $\mathrm{CH}$ : disons que le recours à la représentation permet de penser. L'idée c'est de convoquer des
Questions Vives, $\mathrm{N}^{\circ} 32 \mid 2019$ puestions Vives, $\mathrm{N}^{\circ} 32 \mid 2019$
objets pour penser qui nous intéressent. Les objets qui nous intéressent pour penser par la représentation doivent être les nombres. C'est ça que tu vois après en l'écrivant et en sollicitant le nombre. Quand on est dans le numérique, c'est l'idée de « contenu dans » qui est travaillée, qui est intéressante et qui signifie l'écart. 
Commentaires :

La professeure Flo s'est appuyée sur le film d'une professeure de classe d'étude (Jos). Jos avait amené ses élèves à désigner mathématiquement la différence en faisant étudier les divers écrits produits. Flo l'adapte à la situation des trains : à partir des nombres $7 ; 5$ et 2 , représenter symboliquement la situation par les écritures $5+2=7$ ou $7-5=2$. Cette situation où on trouve un nombre pour en atteindre un autre, en appui sur la représentation visuelle, est bien comprise par les élèves. Pour le chercheur, la situation aurait pu être traitée aussi par la décomposition en $5+2$ du train de 7 wagons, dans le sens que 5 est contenu dans 7 donc l'écart entre 5 et 7 est de 2. Les professeures (Flo, Agn, Emm) soulignent que les élèves ont du mal à appréhender le «contenu dans ». En effet, une question technologique sous-jacente est à appréhender : ajouter une valeur pour atteindre une autre valeur correspond à une technique de composition, trouver une valeur dans une autre correspond à la mise en œuvre du principe inverse. Le signe « = ", pris dans le sens d'équivalence, engage les élèves à une pensée algébrique. L'écriture $7=5+2$ en est le modèle (c'est à dire une représentation qui vise la compréhension des relations entre objets). La boîte et le schéma-ligne portent aussi ces deux situations mais la connaissance des différentes décompositions d'un nombre à deux termes est nécessaire.

L'usage de "contenu dans » ne semble pas facile à comprendre pour les élèves. Par exemple, en fin de réunion, un professeur signale : « beaucoup d'élèves étaient dans le contrat, il y a un 9 au tableau, je sais que $5+4=9$ donc j'écris $5+4=9$ alors qu'on comparait le 7 et le $9 »$ (Tdp 851). Il signale ainsi que les élèves n'ont pas forcément la connaissance utile pour trouver une différence suivant la notion de "contenu dans". En effet, il aurait fallu que ces élèves décomposent 9 en $7+2$. C'est la recherche d'une commune mesure entre deux grandeurs, que nous avions formulée précédemment par l'expression « rendre des termes identiques » (rendre le 9 identique au 7 ; le nombre en plus, 2, étant l'écart). L'hypothèse que souhaite mettre en place concrètement le chercheur porte sur ce travail, avec et dans les nombres : ne plus se situer seulement dans l'expression d'une situation visualisée mais au sein même du domaine numérique. Cependant il ne peut que supposer des pistes de mise en œuvre. Pour donner sens à cette formule abstraite du chercheur («nombre convoqué pour répondre à la situation »), le collectif aura à construire une "ascension de l'abstrait au concret $»^{12}$. Qu'est-ce que cela signifie alors, pour les membres, dans la praxis didactique?

Ainsi commence à se dessiner une synergie entre une conception phénoménologique de l'écart (avec des situations de référence pour visualiser la différence), une conception pragmatique (comment on peut désigner la différence) et une conception algébrique (avec l'utilisation de la décomposition). Cette synergie constituera un fait d'expérience pour le collectif : elle crée un rapport de modélisation permis par l'écriture additive pour désigner la différence.

37 L'enquête du collectif sur la mise en œuvre de la notion de différence est bien amorcée durant cette année scolaire 2012-13 mais elle n'est pas terminée, comme nous allons le voir.

\section{Mise en œuvre dans une classe}

Nous montrons maintenant, en effet, que l'enquête se poursuit pour une de ces professeurs (Flo) sur le point discuté lors du dialogue précédent. Nous présentons un moment d'échanges entre cette professeure et ses élèves. La séance a lieu en décembre 2013, donc l'année scolaire d'expérimentation suivante. La professeure va produire des 
actions qu'elle considère comme nécessaires pour mettre en place la notion de " contenu dans".

Elle a mis en place un rituel : régulièrement, un nombre est proposé aux élèves et doit être décomposé de manières différentes (dans l'exemple ci-dessous, « 8 »). Chaque élève a la possibilité d'écrire ce qu'il sait. Quelques-unes des productions sont ensuite recensées au tableau, donnant ainsi à voir aux autres élèves des décompositions possibles. Des liens sont établis par les élèves entre les écritures; la professeure les montre par un système de signes. En voici la capture d'image dans la figure 6 :

Figure 7 : Tableau de la mise en œuvre de « contenu dans »

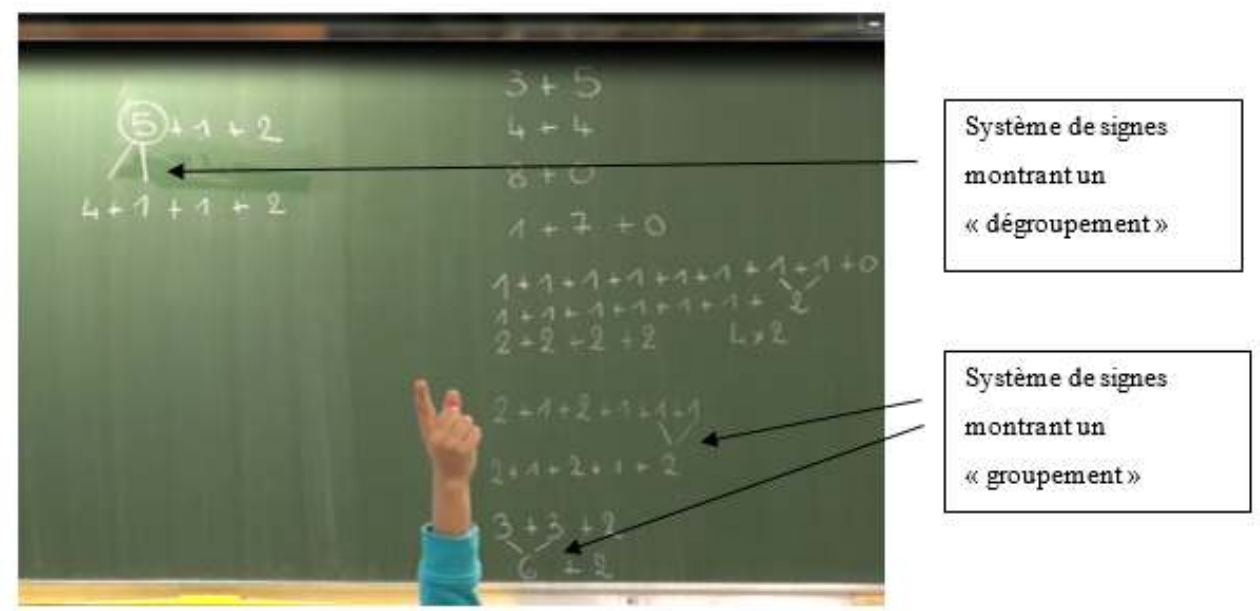

Les écritures proposées par les élèves ont été reportées au tableau; il n'y a aucune recherche d'exhaustivité. Quand un élève remarque un lien entre des écritures, la professeure le note. Par exemple, au bas du tableau, 3 et 3 font 6 donc $« 3+3+2 »$ est équivalent à « $6+2 »$.

Ensuite, elle introduit un nouvel usage de la décomposition (en haut, à gauche sur le tableau) :

Professeure : ce nombre-là (montre $5+1+2)$ c'est 8 . Si je vous demande de l'écrire avec un 4.

Elève $\mathrm{A}: 4+4$

Prof : on peut l'écrire $4+4$. Mais je veux garder le 1 , et le 2 . Donc je vais garder le 1 et le 2 (recopie 1 et 2 ) et je veux voir un 4 . On peut regarder ici, dans le soleil de quoi [photo ci-dessous]?

Elève $B: 4+1$.

Prof : (entoure le 5) je dois dégrouper le 5. Je vais voir dans le soleil du 5 et je cherche les 4.

Elève $\mathrm{A}: 4+1$

Elève $C$ : ou $2+3$ 


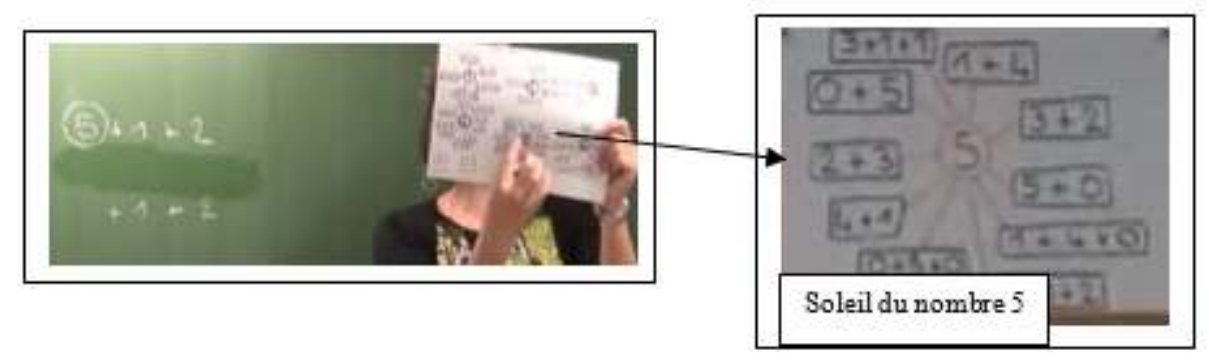

Prof : est-ce qu'il y a 4 dans $2+3$ ? On a dit qu'on voulait le 4 . Le 5 on le dégroupe et on peut écrire $4+1$ à la place (écrit $4+1$ ).

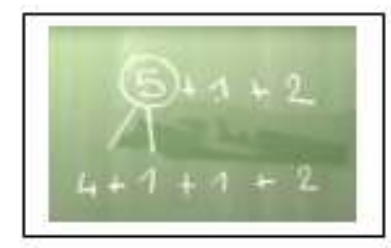

Elève $B$ : ou $1+4$

Prof : vous voyez à quoi il peut servir le soleil ?

Elève $\mathrm{D}:$ et même $4+1+1+2$ c'est égal à 8 .

Professeur : c'est exactement ça. On écrit les différentes écritures de 8.

(Extrait séance CP du 12 décembre 2013)

La professeure a ainsi produit un geste d'enseignement en assumant l'analyse des écritures tout en s'appuyant sur les connaissances des élèves. Le « soleil des nombres " n'est pas un simple répertoire additif. C'est un instrument qui participe à la découverte («vous voyez à quoi il peut servir le soleil ?»), par des moyens expérimentaux, des écritures additives équivalentes.

L'enquête pour une mise en œuvre de la notion de différence, et sur la recherche d'une commune mesure entre deux grandeurs, n'est pas encore conclue mais une concrétisation avec des gestes d'enseignement possibles a été éprouvée. La production d'écritures désignant un nombre, avec utilisation du soleil des nombres et de ses gestes afférents, est diffusée au sein du collectif. Le travail des hypothèses d'ingénierie et la responsabilité de leur validation sont sis dans le « territoire » des professeurs.

\section{Résultats et conclusion}

Le dialogue entre les professeurs et le chercheur dévoile l'enquête qu'ils mènent conjointement pour l'introduction d'une nouvelle notion. Le travail commun consiste (i) à partager les connaissances acquises au fur et à mesure de la mise en place des séquences d'enseignement et (ii) à produire une analyse collective de cette mise en œuvre qui fait milieu pour l'enquête. Cette analyse de l'expérience vécue génère à son tour des connaissances et affermit le style de pensée qui permet au collectif de partager des mêmes concepts et pratiques. Un tel travail est rendu possible par le document support de l'ingénierie et la mise en œuvre anticipée des classes d'étude. Ce décalage temporel est un point d'appui pour les discussions, à condition que soit mise en œuvre la séquence également dans les classes expérimentales.

Un premier résultat de l'étude de cet exemple emblématique de dialogue d'ingénierie montre une exploration de la dialectique contrat/milieu dans un cadre autre que 
scolaire ou didactique stricto sensu. L'enquête s'y déroule entre le « déjà connu » du début de la mise en œuvre et son actualisation pour résoudre des problèmes soulevés par cette mise en œuvre. Les problèmes qui se posent à ce collectif consistent à faire appréhender le sens mathématique de la différence tout en s'appuyant sur des connaissances déjà construites par les élèves afin de maintenir une continuité dans les apprentissages. La notion de nombre « contenu dans » permise par la décomposition du nombre, sa concrétisation dans les actions didactiques, les nouvelles questions engendrées par cette mise en œuvre témoignent d'une avancée dans l'enquête collective.

Un second résultat se trouve dans la description de la relation entre professeurs et chercheur. Elle n'est pas didactique au sens d'une relation où l'un saurait, notamment quelles sont les connaissances à acquérir et le milieu de l'étude à installer, et les autres apprendraient. Tout au contraire, les membres du collectif apprennent par eux-mêmes, sur la base de leurs habitudes d'action et de leurs connaissances, mais à partir des ignorances que leur rapport à l'autre va leur révéler. Les professeurs ont appréhendé l'enjeu de la séquence. Le chercheur et les professeurs ont découvert des pistes de mises en œuvre rendues concrètes. Tous ont constitué des faits d'expérience relatifs à l'introduction de la notion de différence, faits à la fois pratiques et théoriques. Par exemple, comment mettre en évidence la différence dans les systèmes de représentation et qu'est-ce que cela apporte, comment utiliser la décomposition dans un nouvel usage et au sein d'une référence évolutive des annonces. Nous qualifions cette relation entre les membres de l'ingénierie coopérative de relation épistémique coopérative (Joffredo-Le Brun, Morellato, Sensevy, \& Quilio, 2017). Elle met au jour des savoirs, des praxéologies (Chevallard \& Sensevy, 2014). En effet, le fait de parler d'une pratique d'enseignement particulière et contextualisée au sein d'un collectif crée une manière de parler de cette pratique. Cette manière d'en parler permet la compréhension, le développement, la transmission de cette pratique. C'est en cela qu'un tel dialogue comprend $d u$ didactique.

Nous pensons que le partage des connaissances dans leurs dimensions de savoirs d'expérience et de savoirs théoriques, tel que nous avons pu le montrer dans cet exemple emblématique de dialogue entre chercheur et professeurs, peut contribuer à la conception d'une forme nouvelle d'articulation entre recherche et formation continue.

\section{BIBLIOGRAPHIE}

Artigue, M. (1990). Ingénierie didactique. Recherches en Didactique des Mathématiques, 9(3), 281-308.

Artigue, M. (2011). Enseignement et apprentissage de l'algèbre. In A. Mercier, \& R. Jost (Eds), Actes des auditions du comité scientifique (p. 118-123). Lyon: ENS IFé.

Carraher, D., \& Schliemann, A. D. (2014). Early Algebra Teaching and Learning. In S. Lerman (Ed.), Encyclopedia of Mathematics Education (p. 193-196). Springer. 
Chevallard, Y., \& Sensevy, G. (2014). Anthropological Approaches in Mathematics Education, French Perspectives. In Encyclopedia of Mathematics Education (p. 38-43). New York, London: Springer Dordrecht, Heidelberg. Consulté à l'adresse https://hal.archives-ouvertes.fr/ hal-01139352

Dewey, J. (1993). Logique : la théorie de l'enquête (2e éd.). Paris : Presses universitaires de France.

Fassnacht, C., \& Woods, D. (2001, 2013). Transana (version 2.52) [PC]. Wisconsin Center for Education Research - University of Madison (USA). Consulté à l'adresse http://www.transana.org/

Fleck, L. (2008). Genèse et développement d'un fait scientifique (3e éd.). Paris : Flammarion.

Glock, H.-J. (2003). Dictionnaire Wittgenstein. Paris : Gallimard.

Greslard, D., \& Salin, M.-H. (2011). La collaboration entre chercheurs et enseignants au COREM. Une composante essentielle de la mise à l'épreuve d'une ingénierie didactique. In C. Margolinas, M. Abboud-Blanchard, L. Bueno-Ravel, N. Douek, A. Fluckiger, P. Gibel, F. Vandebrouck \& F. Wozniak (Eds), En amont et en aval des ingénieries didactiques (Vol. 2, p. 263-282). Grenoble : La pensée sauvage.

Joffredo-Le Brun, S., Morellato, M., Sensevy, G., \& Quilio, S. (2017). Cooperative Engineering as a Joint Action. European Educational Research Journal. En ligne : http://journals.sagepub.com/doi/ abs/10.1177/1474904117690006.

Kuhn, T. (1974). Second Thoughts on Paradigms. In F. Suppe (Ed.), The Structure of Scientific Theories (p. 459-482). Urbana : University of Illinois Press.

Morellato, M. (2017). Travail coopératif entre professeurs et chercheurs dans le cadre d'une ingénierie didactique sur la construction des nombres : conditions de la constitution de l'expérience collective. Thèse de doctorat. Université de Bretagne occidentale, Rennes, France.

Quilio, S. (2008). Contribution à la pragmatique didactique. Une étude de cas dans l'enseignement des nombres rationnels et décimaux à l'école primaire. Thèse de doctorat. Université de Provence, AixMarseille, France.

Quilio, S. (2012). Une forme caractéristique de condition de l'étude dans la réalisation d'une ingénierie didactique en mathématiques à l'école primaire. Education \& Didactique, 6(2), 9-26.

Sensevy, G. (2007). Des catégories pour décrire et comprendre l'action didactique. In G. Sensevy \& A. Mercier (Eds), Agir ensemble. L'action didactique conjointe du professeur et des élèves (p. 13-49). Rennes : Presses universitaires de Rennes.

Sensevy, G. (2011). Le sens du savoir : éléments pour une théorie de l'action conjointe en didactique. Bruxelles : De Boeck.

Sensevy, G., Forest, D., Quilio, S., \& Morales, G. (2013). Cooperative Engineering as a Specific Design-Based Research. ZDM, The International Journal on Mathematics Education, 45(7), 1031-1043.

Sensevy, G., \& Mercier, A. (2007). Agir ensemble. L'action didactique conjointe du professeur et des élèves. Rennes : Presses universitaires de Rennes.

Verschaffel, L., Bryant, P., \& Torbeyns, J. (2012). The inverse principle: Psychological, mathematical, and educational considerations. Educational Studies en Mathematics, 79(3), 327-334. Wittgenstein, L. (2004). Recherches philosophiques. Paris : Gallimard. 


\section{NOTES}

1. Site de la recherche $:$ http://blog.espe-bretagne.fr/ace/

2. Centre d'Observation et de Recherche sur l'Enseignement des Mathématiques

3. Les professeurs de ces classes de l'enseignement ordinaire (c'est-à-dire des classes fonctionnant selon les programmes et règlements en vigueur dans l'institution Éducation nationale) se sont inscrits volontairement dans le dispositif.

4. Le document support de l'ingénierie décrit les séquences d'enseignement-apprentissage. Il a été rédigé, dans une première version, par les chercheurs et quatre professeurs de classes d'étude (professeurs maîtres-formateurs qui ont déjà travaillé avec la recherche). Cette version a été produite à partir d'une proposition succincte des chercheurs, et mise en place dans les classes d'étude en 2011-12. Ce texte de l'ingénierie a vocation à être modifié les années suivantes selon les bilans effectués par le groupe entier.

5. «Fait d'expérience» est la traduction, retenue dans l'édition française, de l'expression «erfahrungstatsache » utilisée par Fleck. Cette expression est composée de «erfahrung " qui signifie l'ensemble des connaissances acquises par l'expérience et de «tatsache », le fait qui a eu lieu dans la réalité.

6. La notion d'exemple emblématique a été introduite en tant que proto-exemple exemplaire. Les exemples exemplaires permettent de poser un problème et de le résoudre au sein d'une communauté grâce à des règles d'analogies entre une réalité complexe et l'exemple (Kuhn, 1974). Les exemples emblématiques leur sont isomorphes, mais ils ne peuvent être institutionnalisés comme tels qu'après une appropriation partagée dans une communauté de recherche. L'usage spécifié d'une telle conceptualisation en didactique a été initialement proposé par Gérard Sensevy (Sensevy, 2011). Cet usage a été travaillé collectivement dans le Séminaire action qui réunit mensuellement des chercheurs de disciplines différentes, œuvrant en didactique dans la perspective de développement d'une théorie de l'action conjointe en didactique.

7. Le collectif Ace-Arithmécole regroupe plusieurs sous-groupes répartis dans les académies d'Aix-Marseille, Lille, Nice, Rennes et Versailles.

8. Le logiciel Transana est doté d'une interface partagée en quatre fenêtres. Trois d'entre elles sont coordonnées pour assister la transcription des échanges et le repérage d'extraits : zones de texte, d'images vidéo et de flux audio. La fonction "gestion des fichiers " apparaît dans la quatrième fenêtre. Cette fonction permet d'organiser le corpus des films numérisés et de ses textes de la transcription attachés, ainsi que de les associer à des documents. Le découpage de la transcription en extraits produit en fait des clips vidéos. Ce découpage se réalise grâce à des marqueurs temporels insérables dans le texte par un raccourci du clavier. Ces marqueurs repèrent le début et la fin du clip.

9. La différence est aussi enjeu de savoir d'une nouvelle situation didactique, découlant de la comparaison d'annonces ( le nombre inconnu »).

10. Les mots-clés "sens mathématique ", "décomposition", «contenu dans", "représentation", «écrit ", "manipulation matérielle» sont associés de manière très importante aux épisodes relatifs à la différence. Une description de cette méthode et de son usage est consultable (Morellato, 2017, chapitres 5 et 6).

11. Durant la réunion une observation critique de trois films, réalisés dans les classes d'étude, a été menée. Ces classes avancent de manière anticipée à la mise en place des séquences dans les classes expérimentales.

12. Le modèle de l'ascension de l'abstrait au concret est travaillé collectivement lors du Séminaire action. Il est encore à l'étude dans ses dimensions à la fois pratique (comment rendre concrète une notion didactique en vue de l'écriture d'un ouvrage collectif) et théorique (présentation de G. Sensevy, le 27 janvier 2014 : « La conquête du concret. Notes pour une épistémologie de l'analogie paradigmatique : après Marx, La Fontaine »). 


\section{RÉSUMÉS}

Nous cherchons à décrire les pratiques de coopération entre professeurs des écoles et chercheurs. Ceux-ci ont œuvré à la constitution et la mise en place conjuguées d'une ingénierie didactique coopérative sur la construction des nombres au début de l'enseignement primaire (élèves de 6 à 8 ans). Ils ont fondé pour cela un collectif, considéré comme une institution au sein du projet de recherche Ace-Arithmécole. Nous avons décrit une telle institution au travers d'un dialogue spécifique, le dialogue d'ingénierie. Nous analyserons un exemple emblématique d'un tel dialogue en nous appuyant sur la théorie de l'action conjointe en didactique et la notion de contrat / milieu. Ainsi nous appréhenderons les transactions entre les membres du collectif dans une perspective didactique. Nous montrerons que c'est au cœur de ces transactions que sont construits des faits d'expérience, c'est-à-dire des faits appris de l'expérience vécue collectivement au cours d'une enquête sur la mise en œuvre. Ainsi chacun est à même de participer à l'élaboration de praxéologies d'ingénierie.

We seek to describe the cooperative practices of a team of teachers and researchers during the implementation of a cooperative didactic engineering on the construction of number concepts for 6-year-old and 8-year-old pupils (first \& second grades). The collective group founded by teachers and researchers are viewed as an institution within the research project Acearithmécole, with a specific dialogue, that of the engineering dialogue of the project. We analyse this dialogue through an emblematic example. We use the Joint Action Theory in Didactics. We refer to the notion of the dialectic of contract / milieu in order to identify the transactions between the collective members from a didactic point of view. We show how experience-facts have been developed by these transactions, that is to say facts that have been learnt from the collective, lived experience. They are the result of a common investigation of the didactic engineering. Thanks to this collective experience, each member is in a position to take on a share of the construction of the engineering knowledge.

\section{INDEX}

Mots-clés : didactique, ingénierie, coopération, mathématiques, faits d'expérience

Keywords : didactic, engineering, cooperation, mathematics, experience-facts

\section{AUTEUR}

\section{MIREILLE MORELLATO}

Membre associée au laboratoire CREAD, EA 3875, Docteur en Sciences de l'Éducation 